\title{
Spontaneous Hematomas in COVID-19 Patients on Low-Molecular-Weight Heparin
}

\author{
Hitesh Taleja Vipin Venugopal Nair Sunanda Yadav \\ Raghavendra Venkatnarayanan Nilanjan Roy Pankaj Rao \\ Department of Surgery, Armed Forces Medical College, Pune, India
}

\section{Keywords}

COVID-19 · Anticoagulants · Hematoma · Surgical

intervention

\begin{abstract}
Background: COVID-19 patients are at increased risk of coagulopathy. This coagulopathy may be due to a severe proinflammatory state (cytokine storm) and/or by viral sepsis. This can sometimes lead to consumption coagulopathy and decreased platelet count, leading to increased risk of bleeding and may manifest like hematomas in atypical locations. These bleeding manifestations may be spontaneous or can be induced by even minor trauma. Cases: It is a single-center retrospective analysis. Four patients with a confirmed diagnosis of COVID-19 depicting increased risk of bleeding manifestations were included. Patients in our study were managed as per guidelines recommended by the Ministry of Health and Family Welfare Directorate General of Health Services, Government of India. Results: All patients were male. The mean age was $56 \pm 18.64$ years. One patient was managed conservatively with discontinuation of anticoagulants, volume resuscitation, and transfusion of blood products. Drainage with incision was done for 2 patients. One was managed with pigtail drainage. Conclusion: The effect of anticoagulants given in therapy and their varied presentations
\end{abstract}

are discussed in this article. The article concludes that we need vigilant observation to identify this complication in the early period, resulting in successful management.

(c) 2021 The Author(s).

Published by S. Karger AG, Basel

\section{Introduction}

COVID-19, first identified in Wuhan, China in December of 2019, has become a worldwide pandemic. COVID-19 patients are at increased risk of developing a hypercoagulable state. This hypercoagulable state may be due to increased presence of pro-inflammatory cytokines. Although hypercoagulability and thrombotic events are common in COVID-19, bleeding may occur at any time during the disease. Several factors make patients with COVID-19 prone to bleeding such as thrombocytopenia, hyperfibrinolytic state, consumption of coagulation factors, and thromboprophylaxis administration of anticoagulants. Cytokine storm, prolonged tissue hypoxia, and direct invasion of affected tissue are other possible causes. To combat the hypercoagulable and pro-thrombotic state, COVID-19 patients are administered with anticoagulants to reduce ischemic risk. Anticoagulation therapy, whenever indicated in COVID-19 patients, can result in bleeding manifestations like hematomas. karger@karger.com www.karger.com/dmj

Karger $\stackrel{\text { ' }}{5}$

BOPEN ACCESS
(C) 2021 The Author(s)

Published by S. Karger AG, Basel

This is an Open Access article licensed under the Creative Commons Attribution-NonCommercial-4.0 International License (CC BY-NC) (http://www.karger.com/Services/OpenAccessLicense), applicable to the online version of the article only. Usage and distribution for commercial purposes requires written permission.
Correspondence to:

Vipin Venugopal Nair, vipinvenugopalnair@gmail.com 
Table 1. Serial values of hematological and biochemical parameters of cases described

\begin{tabular}{|c|c|c|c|c|c|c|c|c|c|c|c|c|}
\hline Parameters & day 1 & day 3 & day 7 & day 1 & day 3 & day 9 & day 1 & day 3 & day 12 & day 1 & day 3 & day 9 \\
\hline Total lymphocytes (× 10\%/cu mL) & 7.80 & 12.2 & 11.8 & 9.1 & 10 & 7.8 & 7.1 & 8.6 & 7.8 & 10.8 & 12.2 & 8.6 \\
\hline Platelet count $\left(\times 10^{9} / \mathrm{L}\right)$ & 101 & 73 & 126 & 1.12 & 1.02 & 1.00 & 2.2 & 2.06 & 2.00 & 168 & 73 & 2.06 \\
\hline International normalized ratio & 1.1 & 1.4 & 1.4 & 0.9 & 0.9 & 1.1 & 4.02 & 1.34 & 1.2 & 1.4 & 1.4 & 1.34 \\
\hline Total bilirubin, mg/dL & 0.4 & 0.6 & 0.6 & 0.6 & 0.8 & 0.8 & 1.4 & 1.2 & 0.8 & 0.5 & 0.6 & 1.2 \\
\hline Alanine aminotransferase, U/L & 16 & 12 & 24 & 59 & 75 & 28 & 59 & 75 & 28 & 31 & 12 & 75 \\
\hline AST, U/L & 28 & 28 & 33 & 82 & 86 & 47 & 82 & 86 & 47 & 35 & 28 & 86 \\
\hline D-dimer, ng/mL & 1.02 & NA & 0.88 & 0.33 & 0.42 & 0.54 & 0.33 & 0.42 & 0.54 & 0.91 & NA & 0.42 \\
\hline C-reactive protein, $\mathrm{mg} / \mathrm{L}$ & 22.3 & 63.4 & 58.0 & 26 & 42 & 40 & 28 & 36 & 22 & 16.0 & 63.4 & 36 \\
\hline
\end{tabular}
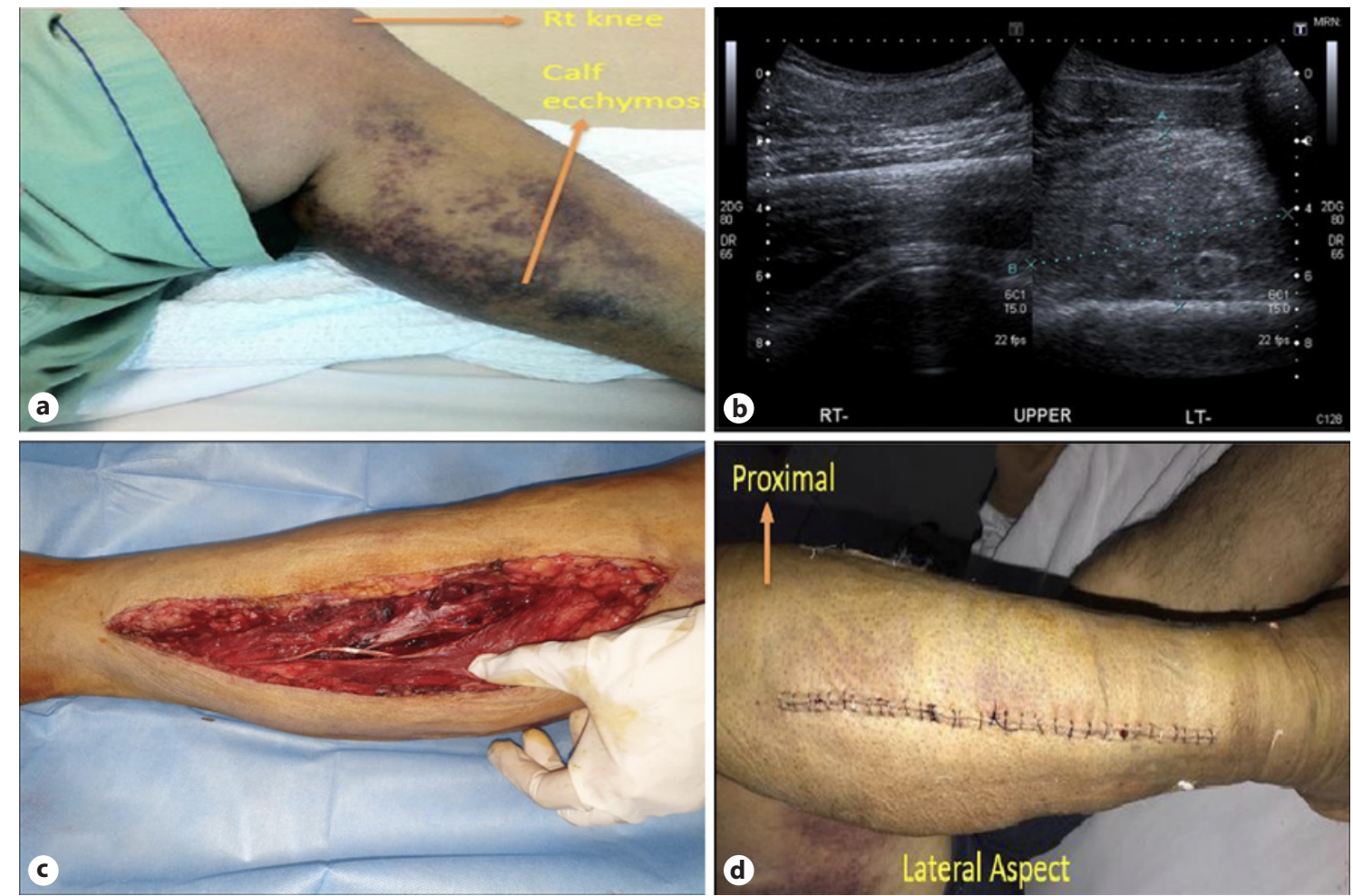

Fig. 1. a Photo showing a tense right calf hematoma with cutaneous ecchymosis. b USG showing $10 \times 5 \mathrm{~cm}$ of hematoma with features of compartment syndrome. c Post fasciotomy wound after evacuation of hematoma. d Delayed primary closure.

\section{Case Report/Case Presentation}

Case 1

A 72-year-old male, with severe COVID-19 infection (RTPCR-positive, was admitted to the emergency department on July 2020. A prophylactic dose of low-molecular-weight heparin (LMWH) of $1 \mathrm{mg} / \mathrm{kg}$ body weight was administered subcutane- ously once daily as per standard guidelines. On post-admission day 20 , the patient developed pain and swelling involving the right calf region. On evaluation, there was tense swelling of $10 \times 8 \mathrm{~cm}$. There was local erythema, tenderness, and pain on passive foot dorsiflexion in the right calf. There was also associated hypo-esthesia on the distribution of deep and superficial peroneal nerves. Distal pulses were palpable in the right lower limb. Serial lab values are append- 

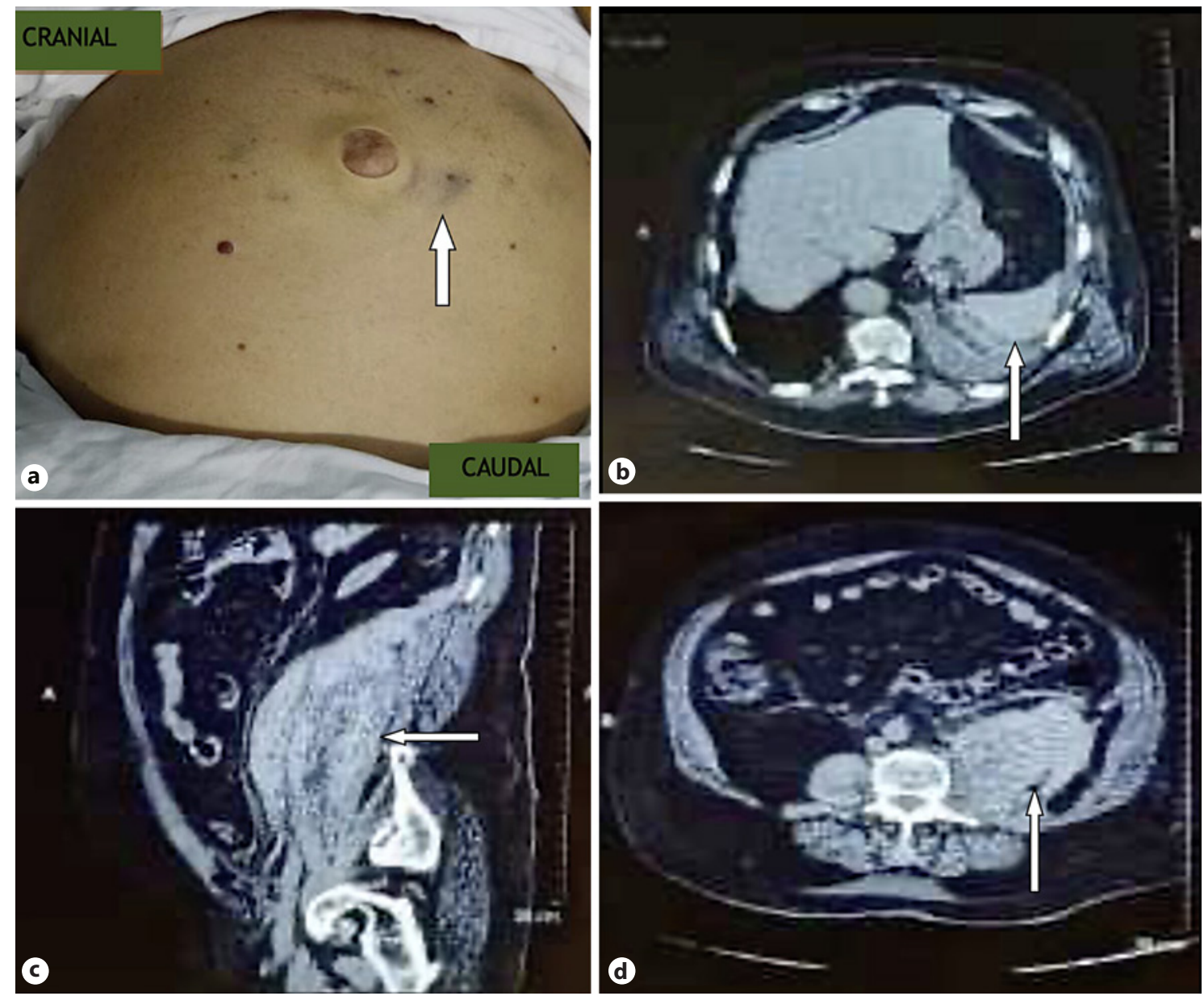

Fig. 2. a Retroperitoneal collection depicting as Cullen's sign. b Transverse CT abdomen showing retroperitoneal collection. $\mathbf{c}$ Sagittal section CT abdomen. d Repeated CT abdomen showing organized and resolving hematoma.

ed in Table 1. Ultrasound of the right calf region showed a $10 \times 5$ $\mathrm{cm}$ collection deep to gastrocnemius muscle with signal characteristics suggestive of a hematoma. The patient underwent fasciotomy of the right lower limb under local anesthesia. Around $200 \mathrm{~mL}$ of hematoma was evacuated. After 3 weeks, the wound was closed. Presently, the patient improved and is ambulant with no functional deficit (shown in Fig. 1).

\section{Case 2}

A 72-year-old male was admitted to the emergency department on August 2020 and tested positive for severe COVID-19 infection. The patient was on prophylactic doses of LMWH as per standard guidelines. On post-admission day 13, the patient developed pain in the left lower abdomen. Serial lab values were appended in Table 1. There was periumbilical ecchymosis, tenderness, and localized guarding in left iliac fossa and left flank. The CT scan abdomen showed $300 \mathrm{~mL}$ of hyperdense collection $(60 \mathrm{HU})$ measuring $10 \times 6 \times 7 \mathrm{~cm}$. This hematoma was located in the retroperitoneum, anterolaterally to the left psoas muscle. The patient was stable and managed conservatively. The hematoma was nonexpanding; the patient was stable. The conservative management in the intensive care facility consisted of volume resuscitation and discontinuation of anticoagulants. Blood and blood products transfusions are given as per requirement. The patient was monitored, with repeated clinical assessment and imaging. The clinical condition improved over time, and there was a complete recovery of the patient (shown in Fig. 2).

\section{Case 3}

A 37-year-old male tested positive for moderate COVID-19 and admitted to the emergency department in September 2020, with a history of trivial trauma to the left thigh. Prophylactic doses of LMWH were initiated as per standard guidelines. The patient developed painful swelling in the left thigh region after 9 days. The swelling gradually progressed over 1 week. It became tense, erythematous, and painful with circumferential involvement of the thigh. Distal pulses were palpable in the left lower limb. Serial lab values are appended in Table 1. An MRI scan of the left thigh region showed hyperintense intramuscular collections at the level of vastus medialis. These collections were extending to involve pelvic, gluteal re- 
Fig. 3. a MRI of the left thigh showing thigh hematoma(left). b Swelling in the left thigh depicted as increase in girth(right).
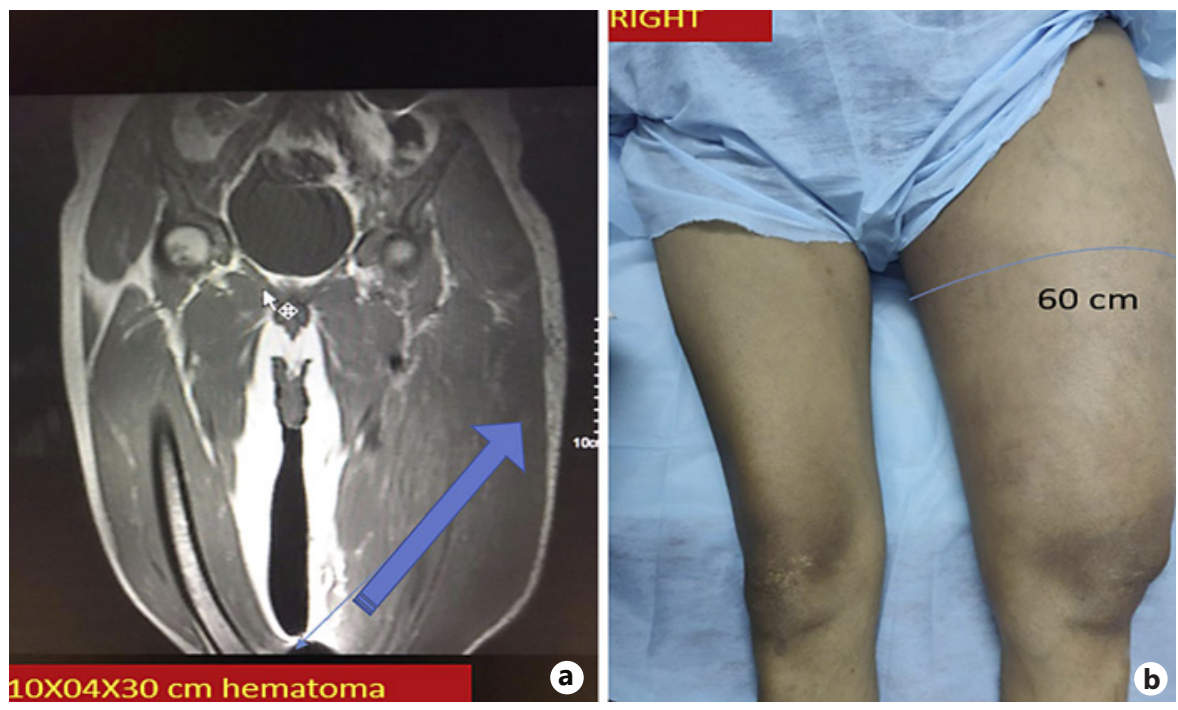

gions, and all compartments of the left thigh. The biggest collection measured was $9.4 \times 3.7 \times 29.3 \mathrm{~cm}$. As the swelling was progressively painful, the patient underwent pigtail drainage of the collection. Two thousand $\mathrm{mL}$ of serous fluid was drained. Presently, the patient is discharged and is under follow-up (shown in Fig. 3).

\section{Case 4}

A 43-year-old male with severe COVID-19 infection was admitted to the emergency care facility in July 2020. The patient was HIVpositive and on antiretroviral drugs. The patient was on prophylactic doses of LMWH as per standard guidelines. The patient developed swelling and ecchymosis on the right side of the chest and medial aspect of the right arm extending to the right elbow region on the fifth day of admission. The patient also developed progressive weakness and numbness in the right upper limb. Evaluation of the right upper limb revealed hypo-esthesia over the distribution of the ulnar, median, and radial nerve. There were features suggestive of musculocutaneous, high ulnar, median, and radial nerve palsy. Serial lab values are appended in Table 1 . A CT scan of the chest axilla and upper limb revealed a $6.5 \times 10.5 \times 10.2 \mathrm{~cm}$ collection in the right axilla. There was intramuscular hematoma of the right pectoralis, right coracobrachialis, and short head of biceps. The patient underwent surgical evacuation of $500 \mathrm{~mL}$ of hematoma of the right pectoral region. Fasciotomy to the right arm was also done because of compartment syndrome in the right upper limb. Intraoperatively, subclavian vessels were displaced inferiorly. The patient was managed in an intensive care facility with repeated clinical assessment and imaging in the post-op period. Over the next 2 weeks, the patient improved with the resolution of symptoms (shown in Fig. 4).

\section{Discussion}

Therapeutic anticoagulation is administered for severe COVID-19 infections. Zhou et al. [1] reported that COVID-19 infection leads to release of pro-inflammatory cy- tokines leading to a hypercoagulable state. This further leads to disseminated intervascular coagulation and venous thromboembolism [1]. In the study of Tang et al. [2] involving 183 patients, significantly elevated fibrin degradation products and $\mathrm{D}$ dimers were reported in $11.5 \%$ of the group with deaths compared to survivors. Miesbach and Makris [3] state that the American Society of Hematology and the International Society on Thrombosis and Haemostasias recommend prophylactic use of LMWH. Manolis states that the microvascular coagulation is seen not only in the microvasculature of lungs but also in various capillary beds of cerebral, cardiac, and peripheral vasculature. The prevalence of venous thromboembolism was around $10-35 \%$ in admitted patients and up to $60 \%$ in autopsies. Pulmonary artery thrombosis without deep vein thrombosis in this study demonstrated the fact that these thromboses inside the pulmonary vessels were formed in situ [4]. The use of prophylactic LMWH was shown beneficial in preventing thrombosis in blood vessels by Cattaneo et al. [5] in another study. This is thought to prevent the microvascular thrombosis in pulmonary vessels, which resulted in respiratory failure [5]. Howev$\mathrm{er}$, due to the use of systemic anticoagulation in COVID-19 patients, bleeding is a significant cause of mortality. All our patients were on prophylactic anticoagulant and manifested spontaneous bleeding in various sites.

Patel et al. [6] reported the first case of COVID-19 patient, who was treated with therapeutic anticoagulants with bleeding manifestation. There was psoas hematoma due to lumbar artery bleeding and managed with arterial embolization [6]. Mattioli et al. [7] reported various atypical hematomas in COVID-19 patients on therapeutic an- 

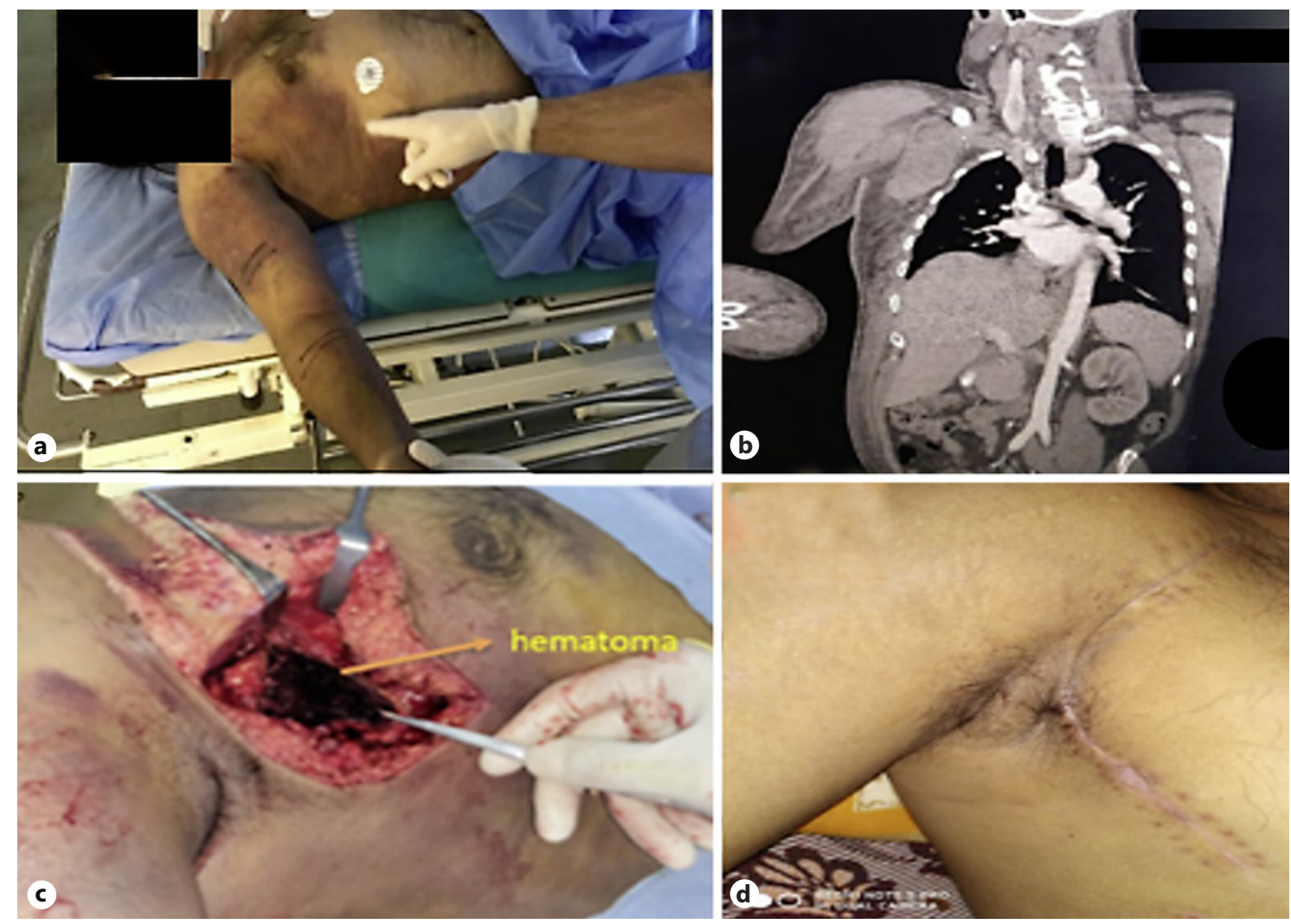

Fig. 4. a On presentation. b CT showing intramuscular and intermuscular hematoma. c Fasciotomy revealing hematoma in the right pectoral region. $\mathbf{d}$ Delayed primary closure.

ticoagulants. This study emphasized the role of anti-factor $\mathrm{X}$ and its importance in understanding plasma heparin levels. Anticoagulant selection needs to be on institutional guidance and dosage titrated with renal status. Renal failure can result in decreased clearance of LMWH and subsequently increase the risk of bleeding. Awareness and a high degree of clinical suspicion are necessary [7]. Dorgalaleh [8] reported fatal GI bleeding, focal hemorrhage in the kidney, and intracranial hemorrhage as initial presentations of COVID-19 patients (not on anticoagulants). This study proposed that prolonged hypoxia causes cell necrosis and mucosal injury, leading to ulceration and bleeding, indicating COVID-19 itself has a high risk for thrombo-hemorrhagic events. Various studies showed the thrombosis- and bleeding-predicting tools that are useful in the management of patients [8]. Al-Samkari et al. [9] showed that elevated D-dimer acts as a significant predictor for bleeding, critical illness, and mortality in COVID-19 patients. In this study conducted with 400 patients admitted due to COVID-19 infection, there was an overall $9.5 \%$ rate of venous thrombosis and also highlighted that there was $4.8 \%$ overall bleeding and 2.4\% major bleeding [9].

Our work highlights hemorrhage as a possible risk factor in patients with moderate to severe COVID-19 infection on anticoagulant treatment. Due to a high rate of coagulopathy among COVID-19 patients, the risk of bleeding should always be considered. In every case, the chances of having concealed bleeding should be looked for during clinical examination. Although rare, a concealed hematoma may be one of the first clinical presentations at the time of diagnosis. This article highlights the fact that all clinicians managing COVID-19 patients on anticoagulants need to be aware of spontaneous bleeding and various methods to manage them.

\section{Conclusion}

The real burden of a hemorrhagic complication resulting from COVID-19 is unknown, and further studies are needed. There is also a requirement for assessing the safe- 
ty of anticoagulant therapies in COVID-19 patients. We recommend that in COVID-19 patients on anticoagulants, clinical suspicion for hematomas needs to be high. When initiating anticoagulation, the drug and dose need to be patient-tailored. Active clinical surveillance can improve the safety of anticoagulation in this subset of individuals.

\section{Statement of Ethics}

This study was cleared by the Hospital Ethical Committee of the Command Hospital, Pune, no 138/20 dated February 18, 2021. Written informed consent for publication was obtained and from all patients. Written consent for photograph and publication of this research has been taken.

\section{Conflict of Interest Statement}

The authors have no conflict of interest.

\section{Funding Sources}

The authors did not receive any funding for this study.

\section{Author Contributions}

V.V.N. designed and wrote the manuscript. H.T. and S.Y. performed the literature review. R.V., N.R., and P.R. reviewed the manuscript. H.T., R.V., and N.R. collected the data and reviewed the manuscript.

\section{Data Availability Statement}

All data generated or analyzed during this case report study are included in this article. Further inquiries can be directed to the corresponding author.

\section{References}

1 Zhou F, Yu T, Du R, Fan G, Liu Y, Liu Z, et al. Clinical course and risk factors for mortality of adult inpatients with COVID-19 in Wuhan, China: a retrospective cohort study. Lancet. 2020 Mar 28;395(10229):1054-62.

2 Tang N, Li D, Wang X, Sun Z. Abnormal coagulation parameters are associated with poor prognosis in patients with novel coronavirus pneumonia. J Thromb Haemost. 2020 Apr; 18(4):844-7.

3 Miesbach W, Makris M. COVID-19: coagulopathy, risk of thrombosis, and the rationale for anticoagulation. Clin Appl Thromb Hemost. 2020 Jan-Dec;26:1076029620938149.
4 Manolis AS, Manolis TA, Manolis AA, Papatheou D, Melita H. COVID-19 infection: viral macro- and micro-vascular coagulopathy and thromboembolism/prophylactic and therapeutic management. J Cardiovasc Pharmacol Ther. 2021 Jan;26(1):12-24.

5 Cattaneo M, Bertinato EM, Birocchi S, Brizio C, Malavolta D, Manzoni M, et al. Pulmonary embolism or pulmonary thrombosis in COVID-19? Is the recommendation to use highdose heparin for thromboprophylaxis justified? Thromb Haemost. 2020 Aug; 120(8): $1230-2$.

6 Patel I, Akoluk A, Douedi S, Upadhyaya V, Mazahir U, Costanzo E, et al. Life-threatening psoas hematoma due to retroperitoneal hemorrhage in a COVID-19 patient on enoxaparin treated with arterial embolization: a case report. J Clin Med Res. 2020 Jul;12(7):458-61.
7 Mattioli M, Benfaremo D, Fustini E, Gennarini S. Atypical spontaneous hematomas in a patient with severe coronavirus disease 2019 (COVID-19). Semin Thromb Hemost. 2020; 46:856-8.

8 Dorgalaleh A. Bleeding and bleeding risk in COVID-19. Semin Thromb Hemost. 2020; 46(7):815-8.

9 Al-Samkari H, Karp Leaf RS, Dzik WH, Carlson JCT, Fogerty AE, Waheed A, et al. COVID-19 and coagulation: bleeding and thrombotic manifestations of SARS-CoV-2 infection. Blood. 2020 Jul 23;136(4):489-500. 